\title{
A SHORT INTERFERING RNA MOLECULAR BEACON FOR THE ATTENUATION OF MYCOBACTERIAL INFECTION
}

\author{
${ }^{1}$ Remo George, ${ }^{1}$ Kathy Nugent, ${ }^{1}$ Norman Bolus, ${ }^{1}$ Joseph Garner, ${ }^{4}$ Jenna Pickering, \\ ${ }^{1}$ Ashley Glaze, ${ }^{1}$ Emma Heard, ${ }^{1,3}$ Jonathan Waugh and ${ }^{1,2}$ M. Tino Unlap \\ ${ }^{1}$ Department of Clinical and Diagnostic Sciences, \\ ${ }^{2}$ Department of Biochemistry and Molecular Genetics, \\ ${ }^{3}$ The Center for Teaching and Learning, \\ University of Alabama at Birmingham, Birmingham, AL USA \\ ${ }^{4}$ Vanderbilt University, Nashville, Tennessee USA
}

Received 2013-12-13; Revised 2014-01-14; Accepted 2014-03-04

\begin{abstract}
The ability of the pathogen Mycobacterium Tuberculosis (MTB) to invade and survive within macrophages of granulomas is attributed to the product of the Mammalian Cell Entry (MCE) operon whose gene, mce4A, encodes a cholesterol transporter that transports host lipids into the bacterium that allows the bacterium to survive during chronic infection. Here, we proposed and tested the hypothesis that a mce $4 A$ siRNA molecular beacon can be used to attenuate mycobacterial infection in macrophages. Mce $4 A$ gene was cloned and expressed in E. coli $(E$. coli-4A) and differentiated U937 cells were transduced with piLenti-siRNA-GFP phage expressing the mce $4 A$ siRNA for $24 \mathrm{~h}$. This was followed by infection with either E. coli-4A or M. smegmatis for $3 \mathrm{~h}$ followed by incubation for $0,3,6,24$ and $48 \mathrm{~h}$. The cells were lysed and the lysates were plated on LB agar plates containing ampicillin $\left(100 \mu \mathrm{g} \mathrm{mL} \mathrm{mL}^{-1}\right)$ or on $7 \mathrm{H} 11$ media and incubated at $37^{\circ} \mathrm{C}$ overnight. Our results showed that the siRNA treatment attenuated E.coli-4A infection in macrophages at 3, 6, 24 and 48 h by 0,77 , 59.6 and $99.7 \%$, respectively. Our results also showed that the siRNA treatment attenuated M. smegmatis infection in macrophages at 3, 6, 24 and 48 h. by 94.8, 70.3, 98.9 and 93.4\%, respectively. In conclusion, a mce $4 A$ siRNA molecular beacon was successfully delivered and stably expressed in macrophages which attenuated $E$. coli expressing $m c e 4 A$ (E. coli-4A) and M. smegmatis infection in macrophages.
\end{abstract}

Keywords: Latent TB, Molecular Beacon, Mce4, Mycobacteria, siRNA

\section{INTRODUCTION}

Latent Tuberculosis (LTB) is a persistent problem in both highly industrialized and developing countries (WHO, 2013; Shea et al., 2014). LTB is characterized by pulmonary granulomas which allow Mycobacterium tuberculosis (Mtb) to survive for years without detection (Barry et al., 2009; Rajni and Meena, 2011). People with LTB are unaware of their condition until their infection becomes acute in about 10-15\% of cases (Mariano, 1995). In the U.S. alone, more than $80 \%$ of Tuberculosis
(TB) cases are from reactivation of LTB infection (Horsburgh and Rubin, 2011). The hallmark of LTB is the granulomas harboring the bacterial infection along with their draining lymph nodes. These granulomas have been shown to possess a necrotic core in the center that provides nutritional source for the persisting Mtb bacteria and is surrounded by concentric layers of macrophages, epitheloid cells, multinucleated Langhans giant cells and lymphocytes (Mariano, 1995; Parasa et al., 2013). These TB lesions are surrounded by highly vascularized tissue (Ulrichs et al., 2005) which enables the targeting of Corresponding Author: Tino Unlap, M., Department of Clinical and Diagnostic Sciences, University of Alabama at Birmingham, Birmingham, AL USA 
latent Mtb with systemically administered drugs. Cases of LTB are on the rise in industrialized nations like U.S owing to the increased transnational migration of populations (Walter et al., 2014). Because of its asymptomatic nature, LTB is difficult to treat (Horsburgh and Rubin, 2011). The treatment regimen for both active and LTB infection consists of an extended course of antibiotics like isoniazid or rifampicin spanning many months which generally carries poor patient compliance rates (Horsburgh and Rubin, 2011). Therefore, because there is lack of efficient treatment for LTB, this study was conducted in order to design a siRNA molecular beacon against one of the mammalian cell entry protein genes that could be used for the attenuation of mycobacterial infection in macrophages.

$M$. tuberculosis survival in granulomas is made possible through its ability to synthesize a set of mammalian cell entry proteins, MCE4 (Arruda et al., 1993). These proteins are encoded on the mce4 operon that consists of five genes designated mce $4 A-F$. During survival of $M$. tuberculosis in the macrophage, these genes are specifically expressed and bioinformatics studies have shown that the products of these genes are cholesterol transporters that help transport lipids from the host macrophage into the mycobacterium allowing the mycobacterium to survive for years during chronic infections (Pandey and Sassetti, 2008). The mce operons are widely distributed throughout the genus Mycobacterium and a non-pathogenic mycobacterial species M. smegmatis, that shares many features with $M$. tuberculosis, possesses a homolog of mce4 (Altschul et al., 1990; Haile et al., 2002; Rathor et al., 2013). Owing to its lower biosafety level restrictions and the presence of an identical MCE4 cholesterol transport system, M. smegmatis (Ms) provides a safe mycobacterial model for preliminary studies (Pelosi et al., 2012) .

Since about $90 \%$ of the TB patients develop LTB and $10 \%$ go on to have acute TB (Kumar and Robbins, 2012), prompt diagnosis and treatment of individuals with LTB is important for the effective control of this disease. Thus, developing a direct Mtb treatment strategy for the asymptomatic latent TB population is vital to our fight against tuberculosis.

Molecular Beacon (MB) siRNA is hairpin shaped single stranded antisense nucleic acid construct with a stem-loop structure. It is postulated that the siRNA stimulates a yet unknown cell surface molecule that initiates the taking up of the siRNA into the host cell via caveolae into caveosomes, which is then transported to the perinuclearly located smooth ER and finally released into the host cell cytoplasm (Erdmann and Barciszewski, 2010). The siRNA is taken up by the bacteria within the host cell and in the presence of the target mce4A mRNA, the loop region of the molecular beacon siRNA hybridizes with the target mRNA. The siRNA-mRNA duplex induces the bacterial interference machinery to cleave the target mRNA and releases the fragments (Van Der Oost and Brouns, 2009; Wiedenheft et al., 2012). The degradation of the mce 4 mRNA ultimately results in depriving the mycobacterium of it energy and carbon sources leading to its loss of virulence and death (Pandey and Sassetti, 2008; Miner et al., 2009; Van Der Geize et al., 2007).

Latent TB is a silent epidemic that threatens the development and fundamental progress of many societies across the globe. In order to counteract this epidemic, effective treatment for TB is critical. To this end, we tested the hypothesis that M. smegmatis infection can be attenuated in macrophages using the siRNA molecular beacon against the mce $4 A$ operon gene that we have previously designed and tested (George et al., 2012). The findings of these studies will demonstrate the utility of attenuating mycobacterial infection using siRNA molecular beacons which can then be easily adapted to eradicating infection in animal models and eventually in humans.

\section{MATERIALS AND METHODS}

\subsection{Mammalian Cell Culture}

U937 human monocytic cells were maintained in RPMI medium supplemented with $10 \%$ fetal calf serum and $50 \mathrm{U} \mathrm{mL}^{-1}$ of penicillin and streptomycin in a $5 \%$ $\mathrm{CO}_{2}$ humidified atmosphere. Cells were passaged at a density of approximately $2 \times 106$ cells $\mathrm{mL}^{-1}$ every other day. Cells were cultured in flasks at $37^{\circ} \mathrm{C}$ for propagation and in 12 well plates with rattail collagen coated glass cover slips for differentiation and infection. U937 cell differentiation was achieved as described previously (Adunyah et al., 1992). Briefly, cells were counted using a hemocytometer and $1 \times 106$ cells were incubated with complete RPMI medium containing 4nM PMA. After $48 \mathrm{~h}$ of treatment with PMA, nonadherent cells were aspirated and fresh media was added prior to initiating treatment.

\subsection{Bacterial Strains and Culture Conditions}

M. smegmatis $m c^{2} 155$ was purchased from ATCC and grown to $\log$ phase $\mathrm{A}_{600}$ of 0.3 in Middlebrook $7 \mathrm{H} 9$ broth (Difco) supplemented with $0.05 \%$ Tween 80 and 
OADC (oleic acid, albumin, glucose, catalase supplement) in $37^{\circ} \mathrm{C}$ with constant shaking at $190 \mathrm{rpm}$. Serial dilutions of the culture were prepared at 1:10, 1:100 and 1:000 in 7H9 media, plated on 7H11 agar plates and incubated at $37^{\circ} \mathrm{C}$. Colonies were counted and the number of Colony Forming Units (CFUs) was determined to calculate the Multiplicity of Infection (MOI) for invasion assay experiments.

E. coli-4A was generated by transforming $E$. coli TOP10® (Invitrogen) with pTrcHis2-TOPO (Invitrogen) containing the mce $4 A$ gene from $M$. smegmatis. E. coli$4 A$ clones were grown on Lennox Broth (LB) containing $100 \mu \mathrm{g} \mathrm{mL} \mathrm{m}^{-1}$ ampicillin and the expression of the recombinant protein was induced by adding Isopropyl- $\beta$ D-thio-Galactoside (IPTG) to the log-phase cultures at a final concentration of $1 \mathrm{mM}$ and grown at $37^{\circ} \mathrm{C}$ with shaking for $5 \mathrm{~h}$. Serial dilutions of the culture were prepared at 1:10, 1:100 and 1:000 in LB media, plated on LB agar plates and incubated at $37^{\circ} \mathrm{C}$. Colonies were counted and the number of Colony Forming Units (CFUs) was determined to calculate the Multiplicity of Infection (MOI) for invasion assay experiments.

\subsection{Cloning of siRNA in piLenti-GFP}

The siRNA target sequence for mce $4 A$ gene was selected using antisense design software provided by Integrated DNA Technologies (IDT). A region of the target sequence spanning nucleotides 5960719-5960747 of $M$. smegmatis coding region (5'TCGGCAGGCTCTCGGGATAGGTGTATCCC-3')

was identified and 4 nucleotides were added to 5 ' end of each strand in order to facilitate the cloning of this fragment into $B b s \quad I$ site of the iLenti-GFP vector (Applied Biological Materials, Inc). This sequence was designed so that upon transcription, the transcript will generate a siRNA with complimentary 5' and 3 ' ends that will form a hairpin structure. This double stranded (ds) fragment was cloned into the iLenti-GFP vector by mixing an aliquot $(3 \mu \mathrm{L})$ of the ds sequence, $4 \mu \mathrm{L}$ of $B b s I$ linearized piLenti-GFP vector, $2 \mu \mathrm{L}$ of $5 \times \mathrm{DNA}$ ligase buffer and $1 \mu \mathrm{L}$ of T4 DNA ligase followed by incubation at room temperature for $2 \mathrm{~h}$. The ligation mix was used to transform E. coli DH5 $\alpha$ cells followed by plating on LB agar plates containing $50 \mu \mathrm{g} \mathrm{mL}$ kanamycin and overnight incubation at $37^{\circ} \mathrm{C}$. Ten colonies were selected and grown overnight in LB broth containing $50 \mu \mathrm{g} \mathrm{mL} \mathrm{mL}^{-1}$ kanamycin. Plasmid isolation from each of the colonies was performed using the SNAP Midiprep kit (Invitrogen) according to the manufacturer's instructions and the plasmids were screened by $E c o R V$ digestion to identify recombinant clones with the correct size and orientation of the insert. $E$. coli containing the insert in the correct orientation was used for lentivirus production.

\subsection{Generation of piLenti-siRNA-GFP Phage}

The piLenti-siRNA-GFP phage was generated in $293 \mathrm{~T}$ cells. $293 \mathrm{~T}$ cells were plated in a $10 \mathrm{~cm}$ tissue culture plate at $90 \%$ confluency. A transfection mix was premade in $2 \mathrm{~mL}$ complete medium supplemented with 0.1mM MEM Non-Essential Amino Acids, 4mM LGlutamine, $1 \mathrm{mM}$ sodium pyruvate, $500 \mu \mathrm{g} \mathrm{mL}$ Geneticin, along with $10 \mu \mathrm{g}$ piLenti-siRNA-GFP vector, $10 \mu \mathrm{g}$ packaging plasmids (Lenti-Combo Mix ${ }^{\circledR}$, Applied Biological Materials, Inc.) and $80 \mu \mathrm{L}$ of lentifectin ${ }^{\circledR}$ transfection reagent (Applied Biological Materials, Inc.), followed by incubation at room temperature for $20 \mathrm{~min}$ and addition of an extra $4.5 \mathrm{~mL}$ serum-free medium. $293 \mathrm{~T}$ cells were transfected with the transfection mix followed by incubation at $37^{\circ} \mathrm{C}$ in a humidified $5 \% \mathrm{CO}_{2}$ incubator. Viral supernatants were harvested after $48 \mathrm{~h}$ of transfection and filtered through $0.45-\mu \mathrm{m}$ PVDF syringe filter (Millipore) followed by concentration using Ultra-Pure ${ }^{\circledR}$ (Applied Biological Materials, Inc.) lentivirus purification kit. Viral titers were determined by RT-PCR using Lentiviral qPCR Titre Kit (Applied Biological Materials, Inc.) according to the manufacturer's instructions.

\subsection{Immunofluorescence}

U937 cells were differentiated with 4nM PMA for 48 $\mathrm{h}$ in a 12 well plate containing rattail collagen coated coverslips at $1 \times 10^{5}$ cells per well. Seeded cells were either transduced or not transduced with piLenti-siRNAGFP construct at a MOI of 5:1 for $24 \mathrm{~h}$. Cells were washed in $1 \times$ PBS, fixed for $15 \mathrm{~min}$ with $4 \%$ Paraformaldehyde (PFA) and mounted on slides using Vectashield ${ }^{\circledR}$ medium containing propidium iodide. Fluorescence imaging was accomplished using a confocal microscope (inverted Nikon TE2000-U microscope equipped with a 60×apochromat oilimmersion TIRFM objective).

\subsection{Western Blot}

Western blot analysis was performed on lysates from differentiated U937 cells which were transduced with the piLenti-siRNA-GFP phage for $24 \mathrm{~h}$, followed by infection with either E. coli-4A or M. smegmatis for $3 \mathrm{~h}$ followed by incubation for $0,3,6,24$ and $48 \mathrm{~h}$. The cells were washed, lysed and the lysate was used 
for Western blot analysis using a GFP monoclonal antibody as previously described (Unlap and Jope, 1997). Specifically, $5.2 \mu \mathrm{g}$ of each protein extract was diluted with Laemmli sample buffer, placed in a boiling water bath for $5 \mathrm{~min}$ and electrophoresed on $10 \%$ SDS-polyacrylamide gel followed by electrotransferring for one hour at $100 \mathrm{~V}$. Subsequent to Western blotting, the nitrocellulose membrane (Pierce) was rinsed in $10 \mathrm{~mL}$ Phosphate Buffered Saline (PBS), pH 7.4. It was then blocked using 10 $\mathrm{mL}$ of Blotto (PBS/5\% low-fat dried milk/0.1\% Tween 20), at $4{ }^{\circ} \mathrm{C}$ with slow shaking for $3 \mathrm{~h}$. Following rinsing with PBS the membrane was slowly shaken overnight with a 1:7500 dilution of rabbit polyclonal anti-GFP antibody (Invitrogen, Inc). The blot was rinsed twice with PBS/0.1\% Tween 20 and then washed three times for $5 \mathrm{~min}$ each using $100 \mathrm{~mL}$ volumes of PBS/0.1\% Tween 20. The membrane was probed for $2 \mathrm{~h}$ at $4{ }^{\circ} \mathrm{C}$ with a 1:5000 dilution of Horseradish Peroxidase (HRP) conjugated goat antirabbit IgG (Invitrogen) in $10 \mathrm{~mL}$ Blotto. Visualization of GFP protein was achieved by the use of an ECL kit for the detection of HRP-labeled antibodies on Western blots (Fisher, Inc). The blot was placed in a film cassette and exposed to X-ray film (HyperfilmECL, GE) and developed.

\subsection{Invasion Assay Time-Course}

PMA differentiated U937 cells were seeded at $2.5 \times 10^{5}$ cells per well in 12 -well plates and incubated for 24h. Seeded cells were either transduced or nottransduced with piLenti-siRNA-GFP construct at a MOI of 5:1 for $24 \mathrm{~h}$. Transduced U937 cells were incubated in fresh medium (HyQ DMEM supplemented with 5\% FCS and $2 \mathrm{mM} \mathrm{L-glutamine)} \mathrm{at} 37^{\circ} \mathrm{C}$ for $30 \mathrm{~min}$ and E.coli- $4 \mathrm{~A}$ or $M$. smegmatis were added to each well at a MOI of 10:1 and incubated at $37^{\circ} \mathrm{C}$ for $3 \mathrm{~h}$. Cells were washed 3 times with HyQ DMEM media which contained 5\% Fetal Bovine Serum (FBS), $1 \%$ penn/strep and $100 \mu \mathrm{g} \mathrm{mL}^{-1}$ kanamycin to remove extracellular bacteria and lysed after 0, 3, 6, 24 and 48h. For lysis, cells were incubated for 10 min in $500 \mu \mathrm{L}$ of lysis buffer $(0.1 \%$ Triton X-100 in PBS, $\mathrm{pH}$ 7.4) and the lysate was plated on LB agar plates containing ampicillin $\left(100 \mu \mathrm{g} \mathrm{mL}^{-1}\right)$ or on $7 \mathrm{H} 11$ media and incubated at $37^{\circ} \mathrm{C}$ overnight. Recombinant E. coli- $4 A$ or M. smegmatis colonies were counted and the numbers of colonies that survived after $0,3,6,24$ and $48 \mathrm{~h}$ post infection was plotted versus time. Infection levels at 3, 6,
24 and $48 \mathrm{~h}$ were compared to that of base line level $(0 \mathrm{~h})$ using Analysis of Variance (ANOVA).

\subsection{Statistical Analysis}

The effect of each treatment was compared between treated and non-treated using ANOVA. A $p$ value $\leq 0.05$ is considered significant.

\section{RESULTS}

\subsection{Cloning of mce4A siRNA in piLenti-GFP Vector}

A region of the mce $4 A$ gene in $M$. smegmatis $M C 155^{2}$ was cloned into the piLenti-GFP vector in order to generate a siRNA against the mce 4 messenger RNA (Fig. 1). The cloning of the $m c e 4 A$ region of the gene allowed the expression of the siRNA with two complementary 5' and 3' ends that will allow the formation of a hairpin structure consisting of a loop, which is the antisense RNA sequence and a stem which is a random sequence that is complimentary between the 5' and 3' ends. The EGFP reporter gene incorporated under the CMV promoter of the piLenti-siRNA-GFP vector allowed the tracking of expressed siRNA in vivo. The successful cloning of the fragment was confirmed by EcoR $V$ restriction digest.

\subsection{Generation of Pilenti-siRNA-GFP Phage}

The generation of the phage was accomplished by transfecting piLenti-siRNA-GFP vector along with packaging plasmids into 293 T cells using lentifectin ${ }^{\circledR}$ transfection reagent (Applied Biological Materials, Inc.). Viral supernatants harvested after $48 \mathrm{~h}$ of transfection were filtered through $0.45 \mu \mathrm{m}$ PVDF syringe filter, concentrated using Ultra-Pure $®$ (Applied Biological Materials, Inc.) lentivirus purification kit and viral titers were determined by RT-PCR using Lentiviral qPCR Titre Kit (Applied Biological Materials, Inc.). The viral titer was found to be $10^{6} \mathrm{IU} \mathrm{mL}^{-1}$.

\subsection{Immunofluorescence}

In order to determine the efficiency of U937 cell transduction with piLenti-siRNA-GFP phage, U937 cells were transduced with the piLenti-siRNA-GFP phage and imaged using a fluorescent microscope. The results show that cells that were not transduced with the piLentisiRNA-GFP phage did not show any green fluorescence while cells that were transduced with the piLenti-siRNAGFP phage showed green fluorescence (Fig. 2). 


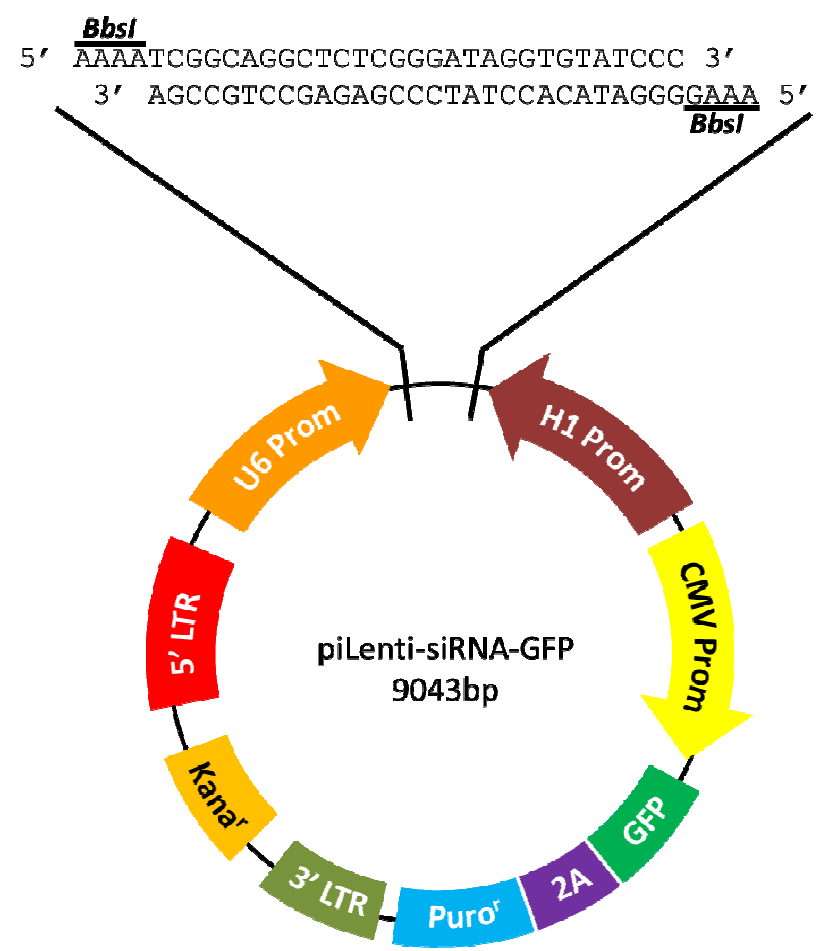

Fig. 1. piLenti-siRNA-GFP vector containing the siRNA molecular beacon construct. A $29 \mathrm{bp}$ region of the M. Smegmatis MC2155 mce4A gene which spans nucleotides 5960719-5960747 was cloned into piLenti-GFP at the BbsI site. Transcription of this fragment is driven by the $\mathrm{U} 6$ promoter and generates a $m c e 4 A$ siRNA.

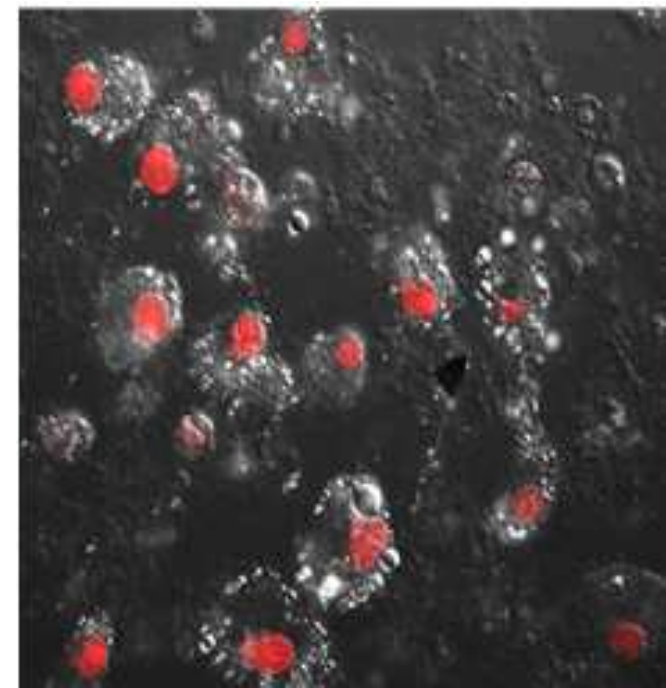

(A)

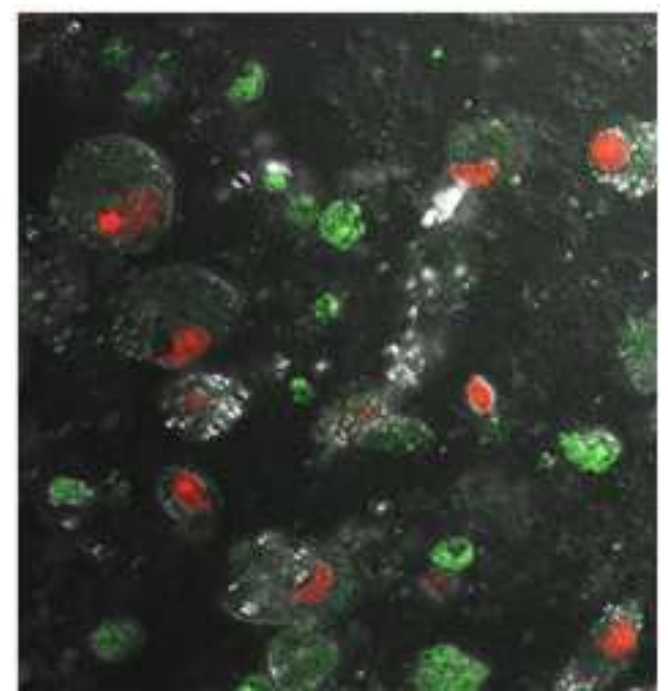

(B)

Fig. 2. Transduced U937 cells express GFP. U937 macrophages were differentiated on rattail collagen coated coverslips for 48 $\mathrm{h}$ with 4nM PMA and were either not transduced (A) or transduced (B) with piLenti-siRNA-GFP construct at a MOI of 5:1 for $24 \mathrm{~h}$. Cells were washed in $1 \times \mathrm{PBS}$, fixed for $15 \mathrm{~min}$ with $4 \%$ PFA and mounted on slides using Vectashield ${ }^{\circledR}$ medium containing propidium iodide followed by fluorescence imaging using a confocal microscope 


\subsection{Western Blot Analysis}

To confirm the expression of the GFP protein, Western blot analysis was performed on lysates from differentiated U937 cells which were transduced with the piLenti-siRNA-GFP phage for $24 \mathrm{~h}$, followed by infection with either E. coli-4A or M. smegmatis for 3 $\mathrm{h}$ followed by incubation for $0,3,6,24$ and $48 \mathrm{~h}$. The cells were washed and lysed and the lysate was used for Western blot analysis using a GFP monoclonal antibody. The results show that the transfected cells constitutively expressed the GFP protein at all of the time points that were examined (Fig. 3).

\subsection{Invasion Assay}

After confirming the expression of the GFP protein by immunofluorescence and Western blot analyses, invasion assay was carried out to determine the effect of

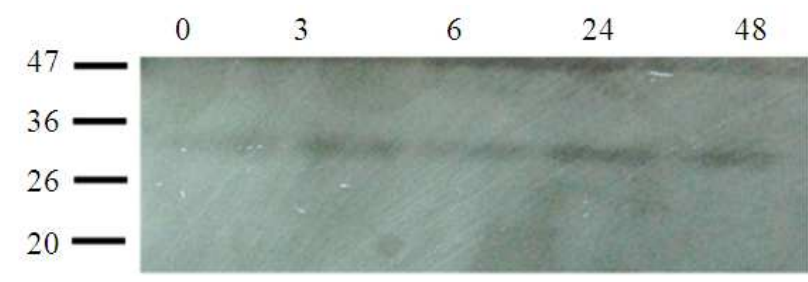

(A) the mce $4 A$ siRNA on macrophage infection. Differentiated U937 macrophages were transduced with piLenti-siRNA-GFP phage for $24 \mathrm{~h}$ followed by infection with E.coli-4A or M. smegmatis for $3 \mathrm{~h}$ and incubated for $0,3,6,24$ and $48 \mathrm{~h}$. The cells were extensively washed and lysed in $0.1 \%$ Triton-X 100 lysis buffer. The lysates were plated on either LB agar containing ampicillin $\left(100 \mu \mathrm{g} \mathrm{mL}^{-1}\right)$ or $7 \mathrm{H} 11$ media for E. coli-4A or $M$. smegmatis, respectively. The degree of attenuation of $E$. coli-4A infection was compared between 3, 6, 24 and $48 \mathrm{~h}$ against that at $0 \mathrm{hr}$ and was found to be $0,77,59.6$ and $99.7 \%$, respectively. The degree of attenuation of $M$. smegmatis infection was compared between 3, 6, 24 and $48 \mathrm{~h}$ against that at $0 \mathrm{hr}$ and was found to be 94.8, 70.3, 98.9 and $93.4 \%$, respectively.

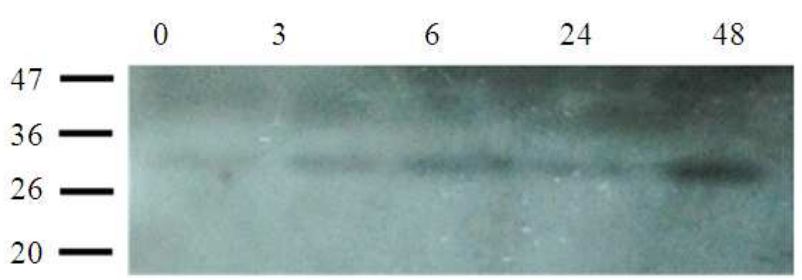

(B)

Fig. 3. The GFP is immunodetected in transduced U937 cells infected with E.coli-4A or M. smegmatis. U937 macrophages were differentiated for $48 \mathrm{~h}$ with $4 \mathrm{nM}$ PMA in 12 -well plates at $1 \times 10^{6}$ cells per well and transduced with piLenti-siRNA-GFP construct at a MOI of 5:1 for $24 \mathrm{~h}$. Transduced U937 cells were infected with IPTG-induced log phase cultures of recombinant $E$. coli-4A or $M$. smegmatis $M C^{2} 155$ at a MOI of 10:1 for $3 \mathrm{~h}$, followed by extensive washing and incubation for $0,3,6,24$ and $48 \mathrm{~h}$. Total cell lysate was obtained at each time point, fractionated on SDS-PAGE and Western blotting was performed using a rabbit polyclonal anti-GFP antibody and HRP conjugated goat anti-rabbit antibody. GFP immunodetection in transduced U937 cells infected with E.coli-4A (A) or M. smegmatis (B) was accomplished using the Immobilon Western Chemiluminescent HRP Substrate (ECL) System ${ }^{\circledR}$ (Millipore). Molecular mass standards, EZ-Run Prestained Protein Marker ${ }^{\circledR}$ (Fisher), are indicated in $\mathrm{k}$

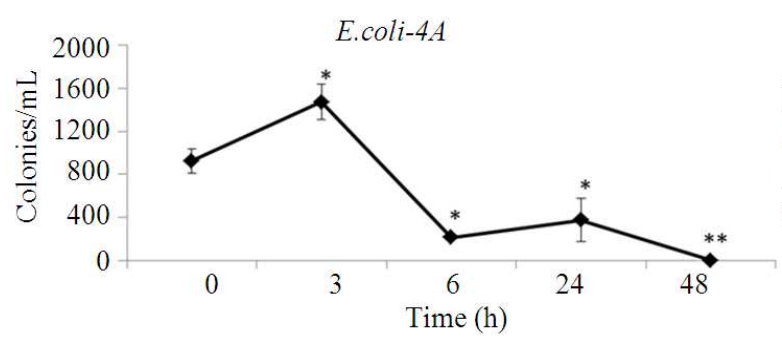

(A)

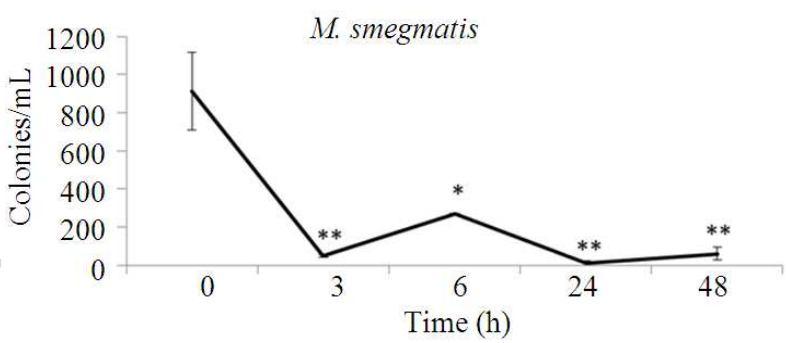

(B)

Fig. 4. Mce4A siRNA attenuates E.coli-4A or M. smegmatis infection in U937 cells. Differentiated U937 cells were transduced at MOI of 5:1 with piLenti-siRNA-GFP lentivirus for $24 \mathrm{~h}$ and were infected with IPTG-induced log phase cultures of recombinant E. coli-4A or $M$. smegmatis $M C^{2} 155$ at a MOI of 10:1 for $3 \mathrm{~h}$, followed by extensive washing and incubation for $0,3,6,24$ and $48 \mathrm{~h}$. Cells were washed extensively in $1 \times \mathrm{PBS}$, lysed in $0.1 \%$ Triton-X 100 lysis buffer and the lysate was plated on $7 \mathrm{H} 11$ agar plates or on LB agar plates containing ampicillin $\left(100 \mu \mathrm{g} \mathrm{mL}{ }^{-1}\right)$ followed by incubation at $37^{\circ} \mathrm{C}$ 
overnight. E.coli-4A (A) and M. smegmatis (B) colonies are counted and the numbers at 3, 6, 24 and 48 h post infection are compared with those at Ohr using ANOVA. $\mathrm{n}=4 ;{ }^{*} \mathrm{p} \leq 0.05 ;{ }^{* *} \mathrm{p} \leq 0.01$

Our results showed that $m c e 4 A$ siRNA attenuated E.coli$4 A$ and $M$. smegmatis infection in macrophages and the degree of attenuation of $E$. coli-4A and M. smegmatis infection was found to be significant ( $\mathrm{p} \leq 0.05)$ (Fig. 4).

\section{DISCUSSION}

The use of molecular beacons and siRNAs for in vivo detection and knockdown of mRNA is gaining popularity. These small hairpin-shaped antisense ribooligonucleotides are stable in cellular environments and bind to their target mRNAs with high specificity (Rhee et al., 2008; Desai et al., 2014). They can easily be modified by the addition of fluorophores and quenchers in order to enhance their utility in detection protocols in vivo (Santangelo et al., 2006; Kim et al., 2008; Bratu et al., 2011; Xue et al., 2011; Hernandez et al., 2014).

Molecular beacon based short interfering RNA (MB siRNA) has also been proven to be a powerful tool for therapeutic gene silencing because of its specificity, broad applicability and high efficiency (Kim et al., 2008; Hong et al., 2010; Ilieva et al., 2013). Small interfering RNA (siRNA) technology has been used to inhibit transcription of hepatitis $G$ virus (Cao et al., 2005), influenza virus (Ge et al., 2003), picorna virus (Lim et al., 2008) and trypanosma brucei (Zhang et al., 2007). SiRNA molecular beacons have been used successfully in the detection and knockdown of telomerase expression in human breast cancer cells (Chang et al., 2007), BMP4 mRNA in hedgehog signaling (Rhee et al., 2008) and aromatase mRNA in breast cancer cells (Zhou et al., 2011). SiRNA technology has been tested successfully for imaging and silencing genes in M. tuberculosis (Harth and Horwitz, 1999; Li et al., 2007) and for inhibiting bacterial growth in human macrophages infected with M. tuberculosis (Harth et al., 2007).

The potential combination of the two technologies, molecular beacon and siRNA, in the health care industry is tremendous. We had previously shown that a siRNA molecular beacon labeled with the fluorophore TYE 665 and quencher Iowa Black RQ-SP could be used for the detection of $M$. smegmatis infection in macrophages (George et al., 2012).

The present study was necessitated by three factors, (1) latent TB continues to be a problem not only for the developing countries but also for industrialized nations like the U.S., (2) because the mycobacterium growth rate is so slow, rapid and specific diagnostic or imaging tests for latent TB are currently not available which naturally leads to (3) there is a lack of specific and efficient treatment for latent TB. To assist in the effort to treat latent TB, this study was conducted in order to test the hypothesis that a molecular beacon siRNA designed against the mce4 operon, which has been shown to be responsible for latent TB infection (Arruda et al., 1993; Saini et al., 2008; Rathor et al., 2013), especially mce4A(Saini et al., 2008; $\mathrm{Xu}$ et al., 2007), could be used for the attenuation of mycobacterial infection. This hypothesis was tested in differentiated U937 cells infected with recombinant $E$. coli expressing $m c e 4 A$ gene or $M$. smegmatis.

In order to design the molecular beacon siRNA, it was first necessary to determine which of the mce4 operon genes conferred the highest degree of virulence to its host. Our previous studies with recombinant $E$. coli expressing various mce 4 operon genes showed that mce $4 \mathrm{~A}$ gene conferred the highest degree of virulence to its host E. coli (George et al., 2012). Because the M. tuberculosis has a slow growth rate and since there is high degree of homology between mce4 operons of $M$. tuberculosis and other mycobacteria (Haile et al., 2002), the mce4A gene of the rapid growing $M$. smegmatis was selected as the target for our siRNA based mycobacterial infectivity studies.

The mce $4 A$ molecular beacon antisense RNA was designed to contain a double stranded stem which consists of nucleotides that are complementary to each other to form a 5-base pair double stranded stem. The loop consists of 29 nucleotides that are complementary to a region of the target mce $4 A$ mRNA. The double stranded stem facilitates cytosolic localization of the siRNA (Chen et al., 2010) in the bacterium and in the presence of the target mce $4 A$ mRNA, the siRNA molecular beacon will bind and degradation of the target mRNA will be induced. The transduction of the molecular beacon siRNA construct using a lentiviral vector expressing GFP (Fig. 1), ensured that the siRNA was constitutively expressed within the differentiated U937 cells throughout the various experimental time points, even up to $48 \mathrm{~h}$ post infection (Fig. 2 and 3 ).

This molecular beacon design tests the rationale that in the absence of the target mce $4 A$ mRNA the molecular beacon remains in its hairpin form while in the presence of its target mRNA the 29 nucleotide loop will compete with the 5 nucleotide stem for hybridization to their respective targets, the loop to the mce $4 A$ mRNA and the stem to its complementary pair on the opposite ends of the molecular beacon. The hybridization of the loop to its target will be greater than that of the strands in the stem, based on the number of nucleotides (29 versus 5). Hybridization of the loop to the mce $4 A$ mRNA will create a temporary siRNAmRNA duplex, which induces the bacterial RNA 
interference machinery () to cleave the target mRNA and release the fragments (Van Der Oost and Brouns, 2009; Wiedenheft et al., 2012). Because the mycobacterium utilizes the product of $m c e 4 A$ for survival on cholesterol for carbon and energy source (Xu et al., 2007; Senaratne et al., 2008; Miner et al., 2009; Rathor et al., 2013), degradation of the mce $4 A$ mRNA will lead to its reduced survival. This study tested the ability of the mce $4 A$ siRNA to attenuate its target mce $4 A$ mRNA in macrophages infected with recombinant E.coli-4A and $M$. smegmatis. The results show that the molecular beacon siRNA construct attenuates its target in macrophages infected with either $E$. coli-4A or M. smegmatis (Fig. 4). Thus, a molecular beacon can be designed against one of the mce4 operon genes in $M$. smegmatis that facilitates the eradication of mycobacterial infection in macrophages.

\section{CONCLUSION}

We have used a GFP expressing lentiviral vector piLenti-siRNA-GFP to successfully deliver and stably express the mce $4 A$ siRNA molecular beacon construct in macrophages infected with either $E$. coli expressing the $m c e 4 A$ gene or $M$. smegmatis. Our results showed that the mce $4 A$ siRNA was able to attenuate E. Coli- $4 A$ and M. smegmatis infection in macrophages.

\section{ACKNOWLEDGEMENT}

The researchers would like to thank Dr. Andrew Gibson and Dr. Robert Kimberly's lab in the Division of Clinical Immunology and Rheumatology at the University of Alabama at Birmingham for donating the U937 cells and other reagents that were used in this study. We would also like to thank the Nuclear Medicine Technology and Clinical Laboratory Science Programs for the support they provided for this study and the Vision Science Research Center for the use of the High Resolution Imaging Facility for the confocal microscopy. George et al. (2012) is a graduate research scholar in the Department of Biochemistry and Molecular Genetics and is supported by two research grants awarded by the American Society of Radiological Technology Research Foundation (Seed grant award\# 2008942, Professional grant award\# 419251).

\section{REFERENCES}

Adunyah, S.E., T.M. Unlap, C.C. Franklin and A.S. Kraft, 1992. Induction of differentiation and c-jun expression in human leukemic cells by okadaic acid, an inhibitor of protein phosphatases. J. Cellular Phys., 151: 415-426. DOI: 10.1002/jcp.1041510223
Altschul, S.F., W. Gish, W. Miller, E.W. Myers and D.J. Lipman, 1990. Basic local alignment search tool. J. Mol. Biol., 215: 403-410. DOI: 10.1016/S00222836(05)80360-2

Arruda, S., G. Bomfim, R. Knights, T. Huima-Byron and L.W. Riley, 1993. Cloning of an M. tuberculosis DNA fragment associated with entry and survival inside cells. Science, 261: 1454-1457. DOI: 10.1126/science.8367727

Barry, C.E., H.I. Boshoff, V. Dartois, T. Dick and S. Ehrt et al., 2009. The spectrum of latent tuberculosis: Rethinking the biology and intervention strategies. Nat. Rev. Microbiol., 7: 845855. DOI: $10.1038 /$ nrmicro2236

Bratu, D.P., I.E. Catrina and S.A. Marras, 2011. Tiny molecular beacons for in vivo mRNA detection. Methods Mol. Biol., 714: 141-157. DOI: 10.1007/978-1-61779-005-8_9

Cao, M., H. Ren, P. Zhao, W. Pan and L. Zhao et al., 2005. Small interfering RNA-mediated inhibition of hepatitis $\mathrm{G}$ virus gene expression in human hepatoma cell Huh-7. Sci. China C Life Sci., 48: 61-69. DOI: $10.1360 / 03 y c 0233$

Chang, E., M.Q. Zhu and R. Drezek, 2007. Novel siRNA-based molecular beacons for dual imaging and therapy. Biotechnol. J., 2: 422-425. DOI: 10.1002/biot.200600257

Chen, A.K., O. Davydenko, M.A. Behlke and A. Tsourkas, 2010. Ratiometric bimolecular beacons for the sensitive detection of RNA in single living cells. Nucleic Acids Res., 38: e148-e148. DOI: 10.1093/nar/gkq436

Desai, H.V., I.S. Voruganti, C. Jayasuriya, Q. Chen and E.M. Darling, 2014. Live-cell, temporal gene expression analysis of osteogenic differentiation in adipose-derived stem cells. Tissue Eng. Part A. DOI: 10.1089/ten.TEA.2013.0761

Erdmann, V.A. and J. Barciszewski, 2010. RNA Technologies and Their Applications. 1st Edn., Springer, New York, ISBN-10: 3642121683, pp: 468.

Ge, Q., M.T. McManus, T. Nguyen, C.H. Shen and P.A. Sharp et al., 2003. RNA interference of influenza virus production by directly targeting mRNA for degradation and indirectly inhibiting all viral RNA transcription. Proc. Natl. Acad. Sci. USA, 100: 2718-2723. DOI: 10.1073/pnas.0437841100

George, R., N. Bolus, J. Garner, K. Nugent and M.T. Unlap, 2012. A short interfering RNA (siRNA) molecular beacon for the detection of mycobacterial infection. J. Biotechnol. Biomaterials, 2: $1000147-$ 1000147. DOI: 10.4172/2155-952X.1000147 
Haile, Y., D.A. Caugant, G. Bjune and H.G. Wiker, 2002. Mycobacterium tuberculosis mammalian cell entry operon (mce) homologs in Mycobacterium Other Than Tuberculosis (MOTT). FEMS Immunol. Med. Microbiol., 33: 125-132. DOI: 10.1016/S09288244(02)00302-4

Harth, G. and M.A. Horwitz, 1999. An inhibitor of exported Mycobacterium tuberculosis glutamine synthetase selectively blocks the growth of pathogenic mycobacteria in axenic culture and in human monocytes: Extracellular proteins as potential novel drug targets. J. Exp. Med., 189: 1425-1436. DOI: 10.1084/jem.189.9.1425

Harth, G., P.C. Zamecnik, D. Tabatadze, K. Pierson and M.A. Horwitz, 2007. Hairpin extensions enhance the efficacy of mycolyl transferase-specific antisense oligonucleotides targeting Mycobacterium tuberculosis. Proc. Natl. Acad. Sci. USA, 104: 71997204. DOI: 10.1073/pnas.0701725104

Hernandez, F.J., L. Huang, M.E. Olson, K.M. Powers and L.I. Hernandez et al., 2014. Noninvasive imaging of Staphylococcus aureus infections with a nuclease-activated probe. Nat. Med. DOI: 10.1038/nm.3460

Hong, H., Y. Zhang and W. Cai, 2010. In vivo imaging of RNA interference. J. Nucl. Med., 51: 169-172. DOI: $10.2967 /$ jnumed.109.066878

Horsburgh, C.R. Jr. and E.J. Rubin, 2011. Clinical practice. Latent tuberculosis infection in the United States. N. Engl. J. Med., 364: 1441-1448. DOI: 10.1056/NEJMcp 1005750

Ilieva, M., P. Della Vedova, O. Hansen and M. Dufva, 2013. Tracking neuronal marker expression inside living differentiating cells using molecular beacons. Front Cell Neurosci., 7: 266. Doi: 10.3389/fncel.2013.00266

Kim, Y., D. Sohn and W. Tan, 2008. Molecular beacons in biomedical detection and clinical diagnosis. Int. J. Clin. Exp. Pathol., 1: 105-116.

Kumar, V. and S.L. Robbins, 2012. Robbins Basic Pathology. 9th Edn., Philadelphia, PA. ISBN-13: 978-1437717815

Li, Y., Z. Chen, X. Li, H. Zhang and Q. Huang et al., 2007. Inositol-1-phosphate synthetase mRNA as a new target for antisense inhibition of Mycobacterium tuberculosis. J. Biotechnol., 128: 726-734. DOI: 10.1016/j.jbiotec.2006.12.019

Lim, T., J. Yuan, H.M. Zhang, A. Sall and Z. Liu et al., 2008. Antisense DNA and RNA agents against picornaviruses. Front Biosci., 13: 4707-4725. DOI: $10.2741 / 3034$
Mariano, M., 1995. The experimental granuloma. A hypothesis to explain the persistence of the lesion. Rev. Inst. Med. Trop. Sao Paulo, 37: 161-176. DOI: 10.1590/S0036-46651995000200012

Miner, M.D., J.C. Chang, A.K. Pandey, C.M. Sassetti and D.R. Sherman, 2009. Role of cholesterol in Mycobacterium tuberculosis infection. Ind. J. Exp. Biol., 47: 407-411.

Parasa, V.R., M.J. Rahman, A.T. Ngyuen Hoang, M. Svensson and S. Brighenti et al., 2013. Modeling Mycobacterium tuberculosis early granuloma formation in experimental human lung tissue. Dis. Model Mech. DOI: 10.1242/dmm.013854

Pandey, A.K. and C.M. Sassetti, 2008. Mycobacterial persistence requires the utilization of host cholesterol. Proc. Natl. Acad. Sci. USA, 105: 43764380. DOI: $10.1073 /$ pnas.0711159105

Pelosi, A., D. Smith, R. Brammananth, A. Topolska and H. Billman-Jacobe et al., 2012. Identification of a novel gene product that promotes survival of Mycobacterium smegmatis in macrophages. PLoS One 7: e31788e31788. Doi: 10.1371/journal.pone.0031788

Rajni and L.S. Meena, 2011. Unique characteristic features of mycobacterium tuberculosis in relation to immune system. Am. J. Immunol., 7: 1-8. DOI: 10.3844/ajisp.2011.1.8

Rathor, N., A. Chandolia, N.K. Saini, R. Sinha and R. Pathak et al., 2013. An insight into the regulation of mce4 operon of mycobacterium tuberculosis. Tuberculosis, 93: 389-397. Doi: 10.1016/j.tube.2013.03.007

Rhee, W.J., P.J. Santangelo, H. Jo and G. Bao, 2008. Target accessibility and signal specificity in live-cell detection of BMP-4 mRNA using molecular beacons. Nucl. Acid. Res., 36: e30-e30. DOI: 10.1093/nar/gkn039

Saini, N.K., M. Sharma, A. Chandolia, R. Pasricha and V. Brahmachari et al., 2008. Characterization of Mce4A protein of Mycobacterium tuberculosis: Role in invasion and survival. BMC Microbiol., 8: 200200. DOI: $10.1186 / 1471-2180-8-200$

Santangelo, P., N. Nitin, L. LaConte, A. Woolums and G. Bao, 2006. Live-cell characterization and analysis of a clinical isolate of bovine respiratory syncytial virus, using molecular beacons. J. Virol., 80: 682-688. DOI: 10.1128/JVI.80.2.682-688.2006

Shea, K.M., J.S. Kammerer, C.A. Winston, T.R. Navin and C.R. Horsburgh, Jr., 2014. Estimated rate of reactivation of latent tuberculosis infection in the United States, overall and by population subgroup. Am. J. Epidemiol., 179: 216-225. DOI: 10.1093/AJE/KWT246 
Senaratne, R.H., B. Sidders, P. Sequeira, G. Saunders and K. Dunphy et al., 2008. Mycobacterium tuberculosis strains disrupted in mce3 and mce4 operons are attenuated in mice. J. Med. Microbiol., 57: 164-170. DOI: 10.1099/jmm.0.47454-0

Ulrichs, T., G.A. Kosmiadi, S. Jorg, L. Pradl and M. Titukhina et al., 2005. Differential organization of the local immune response in patients with active cavitary tuberculosis or with nonprogressive tuberculoma. J. Infect. Dis., 192: 89-97. DOI: 10.1086/430621

Unlap, M.T. and R.S. Jope, 1997. Dexamethasone attenuates NF-kappa B DNA binding activity without inducing I kappa B levels in rat brain in vivo. Brain Res. Mol. Brain. Res., 45: 83-89. 10.1016/S0169-328X(96)00240-9

Van der Geize, R., K. Yam, T. Heuser, M.H. Wilbrink and H. Hara et al., 2007. A gene cluster encoding cholesterol catabolism in a soil actinomycete provides insight into mycobacterium tuberculosis survival in macrophages. Proc. Nat. Acad. Sci. USA, 104: 1947-1952. DOI: 10.1073/pnas.0605728104

Van Der Oost, J. and S.J. Brouns, 2009. RNAi: Prokaryotes get in on the act. Cell, 139: 863-865. DOI: 10.1016/j.cell.2009.11.018

Walter, N.D., J. Painter, M. Parker, P. Lowenthal and J. Flood et al., 2014. Persistent latent tuberculosis reactivation risk in United States immigrants. Am. J. Respir Crit. Care Med., 189: 88-95. DOI: 10.1164/rccm.201308-1480OC
WHO, 2013. Globel tuberculosis report 2013. WHO,

Wiedenheft, B., S.H. Sternberg and J.A. Doudna, 2012. RNA-guided genetic silencing systems in bacteria and archaea. Nature, 482: 331-338. DOI: 10.1038/nature10886

Xu, G., Y. Li, J. Yang, X. Zhou and X. Yin et al., 2007. Effect of recombinant Mce $4 A$ protein of mycobacterium bovis on expression of TNF-alpha, iNOS, IL-6 and IL-12 in bovine alveolar macrophages. Mol. Cell Biochem., 302: 1-7. DOI: 10.1007/s11010-006-9395-0

Xue, Y., R. An, D. Zhang, J. Zhao and X. Wang et al., 2011. Detection of survivin expression in cervical cancer cells using molecular beacon imaging: New strategy for the diagnosis of cervical cancer. Eur. J. Obstet Gynecol. Reprod. Biol., 159: 204-208. DOI: 10.1016/j.ejogrb.2011.06.038

Zhang, J.M., J. Guo, X. Tu, Z.H. Shi and J.J. Hao et al., 2007. Protective effect of Huaxia shallot preparation on human umbilical vein endothelial cell injury induced by oxidized low density lipoprotein and its mechanism. Zhong Xi Yi Jie He Xue Bao, 5: 675-680.

Zhou, C., Y. Mao, Y. Sugimoto, Y. Zhang and N. Kanthamneni et al., 2011. SPANosomes as delivery vehicles for small interfering RNA (siRNA). Mol. Pharm. DOI: 10.1021/mp200426h 